\title{
First isolation and whole genome characterization of Porcine Deltacoronavirus from pigs in Peru
}

\author{
Juan More-Bayona ${ }^{1}$, Mercy Ramírez ${ }^{1}$, Ben Hause $^{2}$, Eric Nelson ${ }^{3}$, and Hermelinda Rivera ${ }^{1}$ \\ ${ }^{1}$ Universidad Nacional Mayor de San Marcos \\ ${ }^{2}$ Cambridge Technologies Worthington Minnesota USA 56187 \\ ${ }^{3}$ South Dakota State University Department of Veterinary and Biomedical Sciences
}

November 16, 2021

\begin{abstract}
Porcine Deltacoronavirus is a newly emergent enteric pathogen affecting swine farms worldwide. It has been detected in several countries in Europe, Asia and North America; yet, it has not been reported in South America. In November 2019, an enteric disease outbreak in a pig farm located in San Martin, Peru; was reported along with submission of three intestinal samples from pigs who succumbed to the disease. Samples were processed for molecular detection by qRT-PCR, viral isolation and further sequencing analysis. A taqman-based RT-PCR was performed to differentiate among the most relevant swine enteric coronaviruses described to date. All samples were positive to Porcine Deltacoronavirus with a cycle threshold $(\mathrm{Ct})$ value between 9-14, revealing a high viral load, while testing negative to Porcine Epidemic diarrhea and Transmissible Gastroenteritis viruses. Following detection, viral isolation was performed using PK-15 and Vero cell lines. After 5 days of inoculation, no cytopathic effect was observed. A second blind passage allowed the observation of cytopathic effect on PK-15 cells, while it remained absent in Vero cells. A fluorescence test using an anti-N monoclonal antibody confirmed viral replication. One sample was processed for whole genome sequencing (NGS). In short, raw reads were imported into CLC genomics and assembled de novo. Out of $479 \mathrm{k}$ reads generated from the sample, $436 \mathrm{k}$ assembled into a $25501 \mathrm{bp}$ contig which was $99.5 \%$ identical to a reference Porcine Deltacoronavirus strain from US within the North American phylogroup. Yet, there are relevant differences at the nucleotide and amino acid levels compared to previously described Porcine Deltacoronavirus strains. Altogether, our findings represent the first report of Porcine Deltacoronavirus in South America, its genomic characterization, which provides information of its evolutionary origin. Thus, this study offers new insights into the molecular epidemiology of Porcine Deltacoronavirus infections in the swine industry.
\end{abstract}

First isolation and whole genome characterization of Porcine Deltacoronavirus from pigs in Peru

\section{Short running title: Porcine Deltacoronavirus in Peru}

Juan A. More-Bayona ${ }^{1}$, Mercy Ramirez-Velasquez ${ }^{1}$, Ben Hause $^{3}$, Eric Nelson ${ }^{2}$, Hermelinda RiveraGeronimo $^{1}$

${ }^{1}$ Laboratory of Virology, Faculty of Veterinary Medicine, Universidad Nacional Mayor de San Marcos, Lima, Peru, 15081

${ }^{2}$ Department of Veterinary and Biomedical Sciences, South Dakota State University, Brookings, SD, USA 57007

${ }^{3}$ Cambridge Technologies, Worthington, Minnesota, USA, 56187

Corresponding authors: 
Juan More-Bayona, Laboratory of Virology, Faculty of Veterinary Medicine, Universidad Nacional Mayor de San Marcos, Lima, Peru 15081. Email: jmoreb@unmsm.edu.pe

Mercy Ramirez-Velasquez, Laboratory of Virology, Faculty of Veterinary Medicine, Universidad Nacional Mayor de San Marcos, Lima, Peru 15081. Email: mramirezv@unmsm.edu.pe

\begin{abstract}
Porcine Deltacoronavirus is a newly emergent enteric pathogen affecting swine farms worldwide. It has been detected in several countries in Europe, Asia and North America; yet, it has not been reported in South America. In November 2019, an enteric disease outbreak in a pig farm located in San Martin, Peru; was reported along with submission of three intestinal samples from pigs who succumbed to the disease. Samples were processed for molecular detection by qRT-PCR, viral isolation and further sequencing analysis. A taqman-based RT-PCR was performed to differentiate among the most relevant swine enteric coronaviruses described to date. All samples were positive to Porcine Deltacoronavirus with a cycle threshold (Ct) value between 9-14, revealing a high viral load, while testing negative to Porcine Epidemic diarrhea and Transmissible Gastroenteritis viruses. Following detection, viral isolation was performed using PK-15 and Vero cell lines. After 5 days of inoculation, no cytopathic effect was observed. A second blind passage allowed the observation of cytopathic effect on PK-15 cells, while it remained absent in Vero cells. A fluorescence test using an anti-N monoclonal antibody confirmed viral replication. One sample was processed for whole genome sequencing (NGS). In short, raw reads were imported into CLC genomics and assembled de novo. Out of $479 \mathrm{k}$ reads generated from the sample, $436 \mathrm{k}$ assembled into a $25501 \mathrm{bp}$ contig which was $99.5 \%$ identical to a reference Porcine Deltacoronavirus strain from US within the North American phylogroup. Yet, there are relevant differences at the nucleotide and amino acid levels compared to previously described Porcine Deltacoronavirus strains. Altogether, our findings represent the first report of Porcine Deltacoronavirus in South America, its genomic characterization, which provides information of its evolutionary origin. Thus, this study offers new insights into the molecular epidemiology of Porcine Deltacoronavirus infections in the swine industry.
\end{abstract}

Keywords: Porcine Deltacoronavirus, Peru, diarrhea, whole genome sequencing, PDCoV isolation. Emerging diseases, Veterinary epidemiology

\title{
Introduction
}

Coronaviruses comprise a large group of single-stranded, positive-sense RNA viruses that infect a broad range of species such as avian and mammals, including humans. Coronaviruses belong to the order Nidovirales , family Coronaviridae, subfamilyCoronavirinae. These enveloped viruses are the largest RNA viruses identified to date ranging from 24-32 kb. Members of the subfamily coronavirinae have been recently grouped into four genus as Alphacoronaviruses, Betacoronaviruses , Gammacoronaviruses and Deltacoronaviruses by the International Committee for Taxonomy of Viruses (ICTV) (Lefkowitz et al., 2018; Woo et al., 2010). Interestingly, it appears that the first two groups have originated from bats, while the latter two emerged from wild birds (Woo et al., 2012).

Porcine deltacoronavirus (PDCoV) is an emergent virus that causes gastrointestinal disease such as diarrhea, vomiting, dehydration and death in young piglets representing a major threat to swine industry (Jung et al., 2015; Li et al., 2019; Zhang, 2016; Zhao et al., 2019). Although PDCoV by itself causes enteric disease, co-infections with other coronaviruses such as Porcine Epidemic Diarrhea Virus (PEDV) and Transmissible Gastroenteritis Virus (TGEV) or other viruses are commonly found (Dara et al., 2018; Feng et al., 2017; Marthaler et al., 2014; Niederwerder, 2018; Song et al., 2015). In this context, PDCoV shows indistinguishable clinical signs from other forms of enteric disease such as PED, TGE and Swine Acute Diarrhea Syndrome (SADS). Thus, proper differential diagnostic relies on genetic detection-based assays that offers a highly sensitive and specific method.

PDCoV has a unique genomic organization. Starting from 5'-end, PDCoV has a 5' untranslated region (UTR), replicase (ORF 1a/b), spike (S), envelope (E), membrane (M), non-structural 6 (NS6), nucleocapsid 
(N), NS7 genes and 3'-UTR (Woo et al., 2010; Zhang, 2016). Interestingly, PDCoV lacks ORF3 and NS1, present in other well-known coronaviruses ( $\mathrm{Si}$ et al., 2020). From these genes, S gene encodes a highly glycosylated protein responsible for binding, cell attachment and entry into target cells, and therefore highly immunogenic. Thus, S gene is commonly used for phylogenetic analysis and vaccine development.

The first identification of PDCoV dates back to 2012 in Hong Kong by Woo et al, followed by multiple outbreaks in the US (Homwong et al., 2016; Marthaler et al., 2014; Wang et al., 2014). Later, PDCoV was reported in Canada (Niederwerder, 2018), Korea (Jang et al., 2018; Lee et al., 2016; Lee \& Lee, 2014), Japan (Suzuki et al., 2018), Thailand (Lorsirigool \& Adthakorn, 2017; Saeng-Chuto et al., 2016) and Vietnam (Le et al., 2018; Saeng et al., 2019). More recently, PDCoV was detected in Mexico (Pérez-Rivera, et al., 2019). In South America, coronavirus infections in porcine are commonly reported. For instance, multiple studies revealed that PEDV and TGEV are present in several countries such as Colombia (Piñeros \& Mogollón Galvis, 2015), Ecuador (Barrera et al., 2017) and Peru (Castro-Sanguinetti et al., 2017). However, PDCoV has never been reported in South America. Hence, we report the first isolation and whole genome sequencing of PDCoV in Peru, providing new insights into the molecular epidemiology of this emerging disease in swine.

\section{Materials and methods}

\subsection{Samples and RNA extraction:}

In November 2019, a report of an enteric clinical disease outbreak in a farm located in San Martin, Peru (see Figure 1C) was made by a local veterinary professional to the National animal health authorities (SENASA). The report indicated high morbidity in young piglets. Intestinal samples were submitted by the farm professionals to the Laboratory of Virology in the Faculty of Veterinary Medicine at the Universidad Nacional Mayor de San Marcos in Lima, Peru; for testing and detection of PEDV, TGEV and PDCoV by qRT-PCR. Intestinal content $(1 \mathrm{ml})$ was processed accordingly. In short, samples were diluted in $5 \mathrm{ml}$ of phosphate buffer solution (PBS) and centrifuged at $250 \mathrm{~g}$ for 5 minutes. Following centrifugation, $1 \mathrm{ml}$ of supernatant was processed for RNA extraction using the QIAmp viral RNA mini kit (Qiagen), following manufacturer's specifications.

\subsection{PCR differentiation and detection}

Samples were assayed by qRT-PCR to differentiate three main enteric coronaviruses affecting pigs as PEDV, TGEV and PDCoV. Thus, we used the EZ-PED/TGE/PDCoV MPX 1.1 kit (Tetracore) following manufacturer's specifications.

\subsection{Viral isolation}

For viral isolation, we tested two different cell lines known to support coronavirus replication. We used PK-15 and Vero cells which were plated in 24-well plates at $90 \%$ of confluence. PK-15 cells were kindly provided by Dr. Jhon Pasick from the Canadian Food Inspection Agency (Ontario, Canada) and Vero cells by the Naval Medical Research Unit Six (NAMRU-6). Briefly, $100 \mu \mathrm{l}$ of intestinal content was filtered and treated with TPCK-trypsin $(10 \mu \mathrm{g} / \mathrm{ml})$ to allow ligand cleavage for further virus attachment to cell receptors. Treated samples were inoculated and incubated for $1 \mathrm{~h}$, followed by replacement of maintenance media with trypsin. Cells were incubated for 5 days at $37{ }^{\circ} \mathrm{C}$ with $5 \% \mathrm{CO}_{2}$, with a second blind passage. Cytopathic effect was evaluated daily, and pictures recorded under light microscopy (Leica Microsystems) using a Leica MC170 HD camera (Leica Microsystems). Following isolation, we performed an immunofluorescence test using a PDCoV anti-nucleoprotein monoclonal antibody (SD55-197), kindly provided by Drs. Eric Nelson and Steven Lawson from the South Dakota State University (South Dakota, US), to confirm the presence of the PDCoV.

\subsection{Whole genome sequencing}

Viral RNA was purified from one sample and processed for next generation sequencing (Illumina, Inc), following manufacturer's specifications. Reads were imported into CLC genomics and assembled de novo. Whole genome and ORF 1a/b sequences of PDCoV were obtained from GenBank $(n=23)$ for phylogenetic analysis. 
For analysis based on $\mathrm{S}$ gene, we also included those from Mexico $(\mathrm{n}=27)$. General information of nucleotide sequences used is listed in Table 1.

\subsection{Phylogenetic analysis}

The PDCoV whole genome, ORF $1 \mathrm{a} / \mathrm{b}$ and $\mathrm{S}$ nucleotide sequences in this study, were aligned using ClustalW from MegaX software (Kumar et al., 2018). We used PDCoV genome sequences obtained from GenBank isolated in the US $(\mathrm{n}=13)$, China $(\mathrm{n}=5)$, Japan $(\mathrm{n}=1)$, Vietnam $(\mathrm{n}=2)$ and Thailand $(\mathrm{n}=2)$. We included sequences from Mexico $(n=4)$ for phylogenetic analysis of S gene. A general time reversible (GTR) nucleotide substitution model with gamma distribution among site rate variation was used, with a maximum likelihood estimation model for phylogenetic reconstruction. Bootstrap analysis was carried out on 1000 data sets. The percentage of nucleotide sequence identity was also calculated.

\section{Results}

3.1. Molecular detection confirms the presence of $\mathrm{PDCoV}$ genome in intestinal samples of pigs suffering from an enteric disease outbreak in Peru

We took advantage of a widely used qRT-PCR assay that allows accurate differentiation of most common enteric coronaviruses in swine such as PEDV, TGEV and PDCoV. qRT-PCR results confirmed the presence of PDCoV RNA in all $(n=3)$ samples analysed, which tested negative to PEDV and TGEV. In addition to its qualitative feature, this assay allows relative quantification. Thus, the viral load was quantified through the amount of genetic target of PDCoV amplified during the process. Samples had low Ct values to PDCoV $(\mathrm{Ct}=9-14)$ which indicated a high PDCoV viral load. From these results, we inferred that PDCoV was involved in the enteric disease with high viral titers.

\subsection{PDCoV replicated in PK-15 while Vero cells did not allow PDCoV propagation}

Following PDCoV detection, our objective was to isolate in vitrousing PK-15 and Vero cell lines. Despite the high viral load detected by PCR, we did not see evidence of cytopathic effect during the first 5 days following inoculation in any of the cell line tested. Thus, we performed a second blind passage to evidence viral replication. Interestingly, $48 \mathrm{~h}$ post inoculation, cytopathic effect was observed in PK-15 cell line, but morphological changes in Vero cell line were absent. These findings were also confirmed by qRT-PCR. Within the major cellular changes observed are: pyknosis, cell rounding, monolayer disruption, cell detachment, which correspond to typical coronavirus cytopathic effect previously described (see Figure 1A). Furthermore, the immunofluorescence test in PK-15 infected cells, confirmed the presence of PDCoV (see Figure 1B). Following isolation, a sample was selected and prepared for genome sequencing.

3.3. Whole genome and ORF $1 \mathrm{a} / \mathrm{b}$ sequencing analysis indicates that Peruvian PDCoV isolate originated from an US PDCoV strain

The nucleotide sequence of Peruvian PDCoV isolate, identified as PDCoV/Peru/isolate/2019, was submitted to GenBank under the accession number MT227371. Our Peruvian PDCoV genome follows similar patterns with other PDCoV genome sequences deposited in GenBank. Thus, this isolate is 25,501 nt in length and consists of, excluding the polyA tail: 5'-UTR (1-480 nt), ORF1a/b (481-11368 nt, 11368-19283 nt), S (19265$22747 \mathrm{nt}), \mathrm{E}(22741-22992 \mathrm{nt}), \mathrm{M}$ (22985-23638 nt), NS6 (23638-23922 nt), N (23943-24971 nt), NS7 (24037$24639 \mathrm{nt})$ and 3'-UTR (24972-25501 nt). A graphical representation of the characterized PDCoV isolate is shown in Figure 2.

Phylogenetic analysis has typically been performed using key major genes of any organism of interest. However, this analysis tends to limit the analysis to a certain gene or group genes. Conversely, whole genome sequencing offers a more complete and deeper genetic characterization compared to partial approaches. In our study, we took advantage of next generation sequencing of our PDCoV isolate to track its evolutionary origin. Our results indicated that our Peruvian strain belongs to the North American phylogroup and is closely related to a PDCoV strain from the US isolated in 2015 (99.5\% of nucleotide identity). Genetic distance of the Peruvian PDCoV strain with other PDCoV analysed reveals high similarity between 97.1 
and 99.5\%. Compared to the US strains, the Peruvian PDCoV has a nucleotide identity between 99.45 $99.51 \%$. Percentages range from 98.6 to $98.74 \%$ when compared to the Chinese strains. Finally, nucleotide identity is $97 \%$ and $97.5 \%$ for Thai and Vietnamese strains, respectively. A summary of nucleotide identity is shown in Table 2. Further analysis based on ORF $1 \mathrm{a} / \mathrm{b}$ showed identical topology to the whole genome sequence phylogenetic tree. Altogether, these results indicate that the virus detected in Peru has emerged from a North American ancestor (see Figure 3A and 3B). Similarly, PDCoV protein sequence analysis resembled the topology of the nucleotide analysis.

\subsection{Phylogenetic analysis of Peruvian PDCoV S gene shows close relationship to the Mexican PDCoV within the North American phylogroup}

$\mathrm{S}$ gene is one of the most variable genes among coronaviruses. This is due to its function in cell attachment and viral replication. This high polymorphism makes the $\mathrm{S}$ gene a powerful tool to estimate the evolutionary relationship among virus strains belonging to the same genetic group. Hence, we performed a phylogenetic analysis using the PDCoV S nucleotide sequences publicly available with our Peruvian S nucleotide sequence to evaluate their evolutionary distance (see Figure 4A). Our results indicate that our PDCoV isolate has close relationship to the Mexican PDCoV strains (98.65 - 99.21\% of nucleotide identity) within the North American phylogroup. Similarly, S protein phylogenetic analysis reveal similar evolutionary relationship among strains analysed. Yet, S phylogenetic analysis evidence that Peruvian PDCoV has a certain degree of divergency from the North American phylogroup.

\subsection{Peruvian PDCoV Spike amino acid sequence reveals unique substitutions compared to other PDCoV strains}

As we observed multiple changes at the nucleotide level in the $\mathrm{S}$ gene, we were interested in evaluating whether these changes represent modifications at the protein level. Thus, we found multiple changes in the S protein sequence (see Figure 4B). Most relevant changes are K96R, G552E, A630V and V1052A that represent unique variations compared to other $\mathrm{PDCoV}$ strains. Other amino acid changes have been also found in Chinese strains such as P38L and A137V. We also detected F530L, like the one found in the Vietnamese strains. Furthermore, the Peruvian strains have a Q642K, like those in Vietnamese and Thai strains. These results provide evidence that PDCoV has undergone to unique changes that indicate a degree of genetic diversity in the Peruvian PDCoV strain. Based on previous studies of PDCoV Spike protein characterization (Shang et al., 2018), amino acid substitutions observed here are located randomly across both S protein subunits (S1 and S2) but none of them is located in the RBD region (S1-CTD).

\section{Discussion}

$\mathrm{PDCoV}$ is one of the most recent and relevant coronaviruses of swine industry. It represents a major threat for swine productivity, and it is responsible for large economic losses worldwide. Yet, PDCoV remains poorly studied despite major efforts made recently. In Peru, multiple cases of enteric disease have occurred, however, these cases are not properly addressed and frequently misdiagnosed. Hence, this study represents the first report, isolation and phylogenetic characterization of a Peruvian PDCoV isolate using the whole genome sequence and its major S protein, revealing unique aspects compared to other PDCoV strains.

Our viral isolation findings contrasted with those detected by qRT-PCR. This provided evidence that a large proportion of viral particles were unable to replicate into a cell line support. This has also been reported by others indicating that low viral isolation rates might be attributed to sample degradation and viral viability (Hu et al., 2015). On the other hand, multiple authors have shown that successful viral isolation are due to other factors such as cell line permissibility and enzyme treatment (Yang et al., 2020; Zhao et al., 2019). In our study, it remains unclear whether trypsin concentration $(10 \mu \mathrm{g} / \mathrm{ml})$ might have played a role in the lack of viral replication in Vero cells. Additional studies, at different trypsin concentration, will elucidate Vero cell line permissibility to PDCoV. Interestingly, we did not observe cell toxicity in our assays, as that was absent following the first 5 days of inoculation and PDCoV cytopathic effect was evidenced in the second passage. These results contrast with other claims that cell toxicity is common during PDCoV isolation using intestinal content or fecal samples (Hu et al., 2015). Nevertheless, further studies are required to clarify the 
implications of cell permeability to PDCoV in viral replication and its effects on clinical presentation.

Whole genome analysis revealed that our isolate was closely related to North American strains. The close relationship within the Peruvian and the North American strains indicate they share a common phylogenetic ancestor and revealed that the Peruvian isolate emerged from a US strain. In 2016, Perez-Rivera et al. reported the first phylogenetic analysis of $\mathrm{PDCoV}$ in Mexico, focusing the analysis on the S gene nucleotide sequence, and no report of PDCoV whole genome sequence was made (Pérez-Rivera et al., 2019). Thus, we were unable to evaluate the phylogenetic relationship using the whole genome sequence of PDCoV from Peru and Mexico. We believe that this would add deeper understanding about its appearance in Peru and contribute to its epidemiology in South America.

Due to its high variability, S gene nucleotide sequences have been used to estimate the genetic relationship of PDCoV strains worldwide. Perez-Rivera et al. demonstrated that Mexican PDCoV formed two clades: the group including strains isolated in 2015, and those PDCoV isolated in 2017 (Pérez-Rivera et al., 2019). In our study, phylogenetic analysis using S gene nucleotide sequences reveals that Peruvian PDCoV grouped closely to the Mexican strain isolated in 2015 within the North American phylogroup. This indicates a close relationship among PDCoV strains from Mexico, the US and Peru, sharing a common ancestor and evidencing a dissemination route of PDCOV from North America to South America. Interestingly, multiple non silent mutations were found in the Peruvian PDCoV strain compared to other genomes. Even though some these mutations have been described in other PDCoV strains, some are unique to the Peruvian isolate revealing that this isolate has undergone phenotypical changes after its emergence in North America. Although these substitutions were not located in critical regions of glycosylation sites nor in the RBD region (S1-CTD), they might have influence in ligand/receptor interaction. Further studies are required to clarify whether these modifications have implications in the pathobiology and development of the clinical disease.

To date, it is unclear how PDCoV was introduced in Peru. However, there is a long history of commerce between Peru and North American countries that has expanded in recent years. The National Service of Animal Health in Peru (SENASA) reported the import of a large number of purebred animals ( 150 tons) during the 2014 and 2018 period. Furthermore, Peru imports feed ingredients for swine farms mainly from the US (MINAGRI, 2020). Altogether, this might explain the possible routes for PDCoV entrance into the country, similar to that described for other viruses of importance for swine industry (Dee et al., 2018; Ramírez et al., 2019). Interestingly, there is no report of PDCoV in other South American countries so the introduction from those is unlikely. Nevertheless, further studies are needed to understand the epidemiology of this disease in Peru and its relationship to other countries.

In conclusion, Peruvian PDCoV strain was successfully sequenced, isolated and phylogenetically analysed demonstrating that this isolate has been derived from a US strain. To our knowledge, this is the first report of a PDCoV strain detected in South America and offers new insights about the epidemiology of PDCoV worldwide.

\section{Acknowledgement}

The authors would like to express their gratitude to Drs. Mariluz Arainga-Ramirez, and Steven Lawson for their contribution, and the Universidad Nacional Mayor de San Marcos for its financial support. We also would like to acknowledge the farm professionals and the national health authorities (SENASA-Peru) for the information provided.

\section{Conflict of interest}

All authors have declared no conflict of interest

\section{Ethical statement}

The authors confirm that the ethical policies of the journal, as noted on the journal's author guidelines page, have been adhered to. No ethical approval was required as research samples were obtained in accordance with guidelines from the Peruvian National authorities in animal health. 


\section{Data availability}

The data that support the findings of this study were submitted to the GenBank database (https://www.ncbi.nlm.nih.gov/genbank/) with accession number MT227371 for the Peruvian strain of PDCoV obtained.

\section{References}

Barrera, M., Garrido-haro, A., Vaca, M. S., Granda, D., Acosta-batallas, A., \& Pérez, L. J. (2017). Tracking the Origin and Deciphering the Phylogenetic Relationship of Porcine Epidemic Diarrhea Virus in Ecuador.BioMed Research International , 1-7.

Castro-Sanguinetti, G., Mercy Ramírez, V., Juan More, B., Alberto Manchego, S., \& Hermelinda Rivera, G. (2017). Isolation and molecular detection of emerging porcine epidemic diarrhea virus strains in Lima, Peru. Revista de Investigaciones Veterinarias Del Peru,28 (4). https://doi.org/10.15381/rivep.v28i4.13885

Dara, R., Pasma, M. M. T., \& Poljak, L. M. Z. (2018). Herd-level prevalence and incidence of porcine epidemic diarrhoea virus (PEDV) and porcine deltacoronavirus (PDCoV ) in swine herds in Ontario, Canada.Transboundary and Emerging Diseases , 65 (June 2017), 1197-1207. https://doi.org/10.1111/tbed.12858

Dee, S. A., Bauermann, F. V., Niederwerder, M. C., Singrey, A., Clement, T., de Lima, M., .. Diel, D. G. (2018). Survival of viral pathogens in animal feed ingredients under transboundary shipping models. PLoS ONE , 14 (3), 1-18. https://doi.org/10.1371/journal.pone.0214529

Feng, K. M. J., Li, G. C. D., Bai, L. Z. Y., \& Ma, Q. W. J. (2017). The detection and phylogenetic analysis of porcine deltacoronavirus from Guangdong Province in Southern China. Transboundary and Emerging Diseases , (November 2016), 1-8. https://doi.org/10.1111/tbed.12644

Homwong, N., Jarvis, M. C., Lam, H. C., Diaz, A., Rovira, A., Nelson, M., \& Marthaler, D. (2016). Characterization and evolution of porcine deltacoronavirus in the United States. Preventive Veterinary Medicine, 123 (September 2014), 168-174. https://doi.org/10.1016/j.prevetmed.2015.11.001

Hu, H., Jung, K., Vlasova, A. N., Chepngeno, J., Lu, Z., Wang, Q., \& Saif, L. J. (2015). Isolation and characterization of porcine deltacoronavirus from pigs with diarrhea in the United States. Journal of Clinical Microbiology , 53 (5), 1537-1548. https://doi.org/10.1128/JCM.00031-15

Jang, G., Kim, S., Lee, Y. J., Kim, S., Lee, D. S., Lee, K., \& Lee, C. (2018). Isolation and Characterization of Korean porcine deltacoronavirus strain KNU16-07. Journal of Veterinary Science ,19 (4), 577-581.

Jung, K., Hu, H., Eyerly, B., Lu, Z., Chepngeno, J., \& Saif, L. J. (2015). Pathogenicity of 2 Porcine Deltacoronavirus Strains in Gnotobiotic Pigs. Emerging Infectious Diseases , 21 (4), 650-654.

Kumar, S., Stecher, G., Li, M., Knyaz, C., \& Tamura, K. (2018). MEGA X: Molecular evolutionary genetics analysis across computing platforms. Molecular Biology and Evolution , 35 (6), 1547-1549. https://doi.org/10.1093/molbev/msy096

Le, V. P., Song, S., Hyun, B., Gyu, A., Park, N., \& Thach, N. (2018). A novel strain of porcine deltacoronavirus in Vietnam. Archives of Virology , 163 (1), 203-207. https://doi.org/10.1007/s00705-017-3594-8

Lee, J. H., Chung, H. C., Nguyen, V. G., Moon, H. J., Kim, H. K., Park, S. J., . . Park, B. K. (2016). Detection and Phylogenetic Analysis of Porcine Deltacoronavirus in Korean Swine Farms , 2015. Transboundary and Emerging Diseases , 63 (January 2013), 248-252. https://doi.org/10.1111/tbed.12490

Lee, S., \& Lee, C. (2014). Complete Genome Characterization of Korean Porcine Deltacoronavirus strain KOR/KNU14-04/2014. Genome Announcements , 2 (6), 4-5. https://doi.org/10.1128/genomeA.0119114.Copyright

Lefkowitz, E. J., Dempsey, D. M., Hendrickson, R. C., Orton, R. J., Siddell, S. G., \& Smith, D. B. (2018). Virus taxonomy : the database of the International Committee on Taxonomy of Viruses ( ICTV ). Nucleic 
Acids Research , 46 (October 2017), 708-717. https://doi.org/10.1093/nar/gkx932

Li, B., Zheng, L., Li, H., Ding, Q., Wang, Y., \& Wei, Z. (2019). Porcine deltacoronavirus causes diarrhea in various ages of field-infected pigs in China. Bioscience Reports ,39 (September), 1-10.

Lorsirigool, A., \& Adthakorn, K. S. (2017). The genetic diversity and complete genome analysis of two novel porcine deltacoronavirus isolates in Thailand in 2015. Virus Genes , 53 (2), 240-248. https://doi.org/10.1007/s11262-016-1413-z

Marthaler, D., Jiang, Y., Collins, J., \& Rossow, K. (2014). Complete Genome Sequence of Strain SDCV/USA/Illinois121/2014, a Porcine Deltacoronavirus from the United States. Genome A , 2 (2), 1-2. https://doi.org/10.1128/genomeA.00218-14.Copyright

Marthaler, D., Raymond, L., Jiang, Y., Collins, J., Rossow, K., \& Rovira, A. (2014). Rapid detection, complete genome sequencing, and phylogenetic analysis of porcine deltacoronavirus. Emerging Infectious Diseases , 20 (8), 1347-1350. https://doi.org/10.3201/eid2008.14-0526

MINAGRI. (2020). Anuario estadistico de Comercio Exterior Agrario 2019, 77. Retrieved from https://books.google.com/books?id=uacSAQAAQBAJ\&pgis=1

Niederwerder, M. C. (2018). Swine enteric coronavirus disease : A review of 4 years with porcine epidemic diarrhoea virus and porcine deltacoronavirus in the United States and Canada. Transboundary and Emerging Diseases , 65 (August 2017), 660-675. https://doi.org/10.1111/tbed.12823

Perez-Rivera, C., Ramirez-Mendoza, H., Mendoza-Elvira, S., Segura-Velazquez, R., \& Sanchez-Betancourt, J. I. (2019). First report and phylogenetic analysis of porcine deltacoronavirus in Mexico. Transboundary and Emerging Diseases , 66 (4), 1436-1441. https://doi.org/10.1111/tbed.13193

Pineros, R., \& Mogollon Galvis, J. (2015). Coronavirus in Pigs: Significance and Presentation of Swine Epidemic Diarrhea Virus (PEDV) in Colombia. Revista de Medicina Veterinaria , (29), 73-89.

Ramirez, M., Bauermann, F. V., Navarro, D., Rojas, M., Manchego, A., Nelson, E. A., .. Rivera, H. (2019). Detection of porcine reproductive and respiratory syndrome virus (PRRSV) 1-7-4-type strains in Peru. Transboundary and Emerging Diseases , 66 (3), 1107-1113. https://doi.org/10.1111/tbed.13134

Saeng-Chuto, K., Lorsirigool, A., Temeeyasen, G., Vui, D. T., Stott, C. J., Madapong, A., .. Nilubol, D. (2016). Different Lineage of Porcine Deltacoronavirus in Thailand, Vietnam and Lao PDR in 2015. Transboundary and Emerging Diseases , 1-8. https://doi.org/10.1111/tbed.12585

Saeng, K., Christopher, J., Dam, J. S., Vui, T., Tantituvanont, A., \& Nilubol, D. (2019). Retrospective study, full - length genome characterization and evaluation of viral infectivity and pathogenicity of chimeric porcine deltacoronavirus detected in Vietnam. Transboundary and Emerging Diseases , (August), 1-16. https://doi.org/10.1111/tbed.13339

Shang, J., Zheng, Y., Yang, Y., Liu, C., Geng, Q., Tai, W., .. Zhang, W. (2018). Cryo-Electron Microscopy Structure of Porcine Deltacoronavirus Spike Protein in the Prefusion State. Journal of Virology , 92 (4), $1-14$.

Si, F., Hu, X., Wang, C., Chen, B., Wang, R., Dong, S., .. Li, Z. (2020). Enhances Viral Proliferation by Inhibiting Apoptosis of Infected Cells. Viruses , 12 (214), 1-18.

Song, D., Zhou, X., Peng, Q., Chen, Y., Zhang, F., Huang, T., ... Tang, Y. (2015). Newly Emerged Porcine Deltacoronavirus Associated With Diarrhoea in Swine in China: Identification, Prevalence and Full-Length Genome Sequence Analysis. Transboundary and Emerging Diseases ,6 (M), 575-580. https://doi.org/10.1111/tbed.12399

Suzuki, T., Shibahara, T., Imai, N., Yamamoto, T., \& Ohashi, S. (2018). Genetic characterization and pathogenicity of Japanese porcine deltacoronavirus. Infection, Genetics and Evolution , \#pagerange\#. https://doi.org/10.1016/j.meegid.2018.03.030 
Wang, L., Byrum, B., \& Zhang, Y. (2014). Detection and Genetic Characterization of Deltacoronavirus in Pigs, Ohio, USA, 2014.Emerging Infectious Diseases, 20 (7), 1227-1230.

Woo, P. C. Y., Huang, Y., Lau, S. K. P., \& Yuen, K. (2010). Coronavirus Genomics and Bioinformatics Analysis. Viruses , 2 , 1804-1820. https://doi.org/10.3390/v2081803

Woo, P. C. Y., Lau, S. K. P., Lam, C. S. F., Lau, C. C. Y., Tsang, A. K. L., Lau, J. H. N., . . Yuen, K. (2012). Discovery of Seven Novel Mammalian and Avian Coronaviruses in the Genus Deltacoronavirus Supports Bat Coronaviruses as the Gene Source of Alphacoronavirus and Betacoronavirus and Avian Coronaviruses as the Gene Source of Gammacoronavirus and Deltacoronavi. Journal of Virology ,86 (7), 3995-4008. https://doi.org/10.1128/JVI.06540-11

Yang, Y., Meng, F., Qin, P., Herrler, G., \& Huang, Y. (2020). Trypsin promotes porcine deltacoronavirus mediating cell-to-cell fusion in a cell type- dependent manner. Emerging Microbes and Infections ,9 , 457468. https://doi.org/10.1080/22221751.2020.1730245

Zhang, J. (2016). Porcine deltacoronavirus : Overview of infection dynamics, diagnostic methods, prevalence and genetic evolution. Virus Research , 226 , 71-84. https://doi.org/10.1016/j.virusres.2016.05.028

Zhao, Y., Qu, H., Hu, J., Fu, J., Chen, R., Li, C., .. Huang, X. (2019). Characterization and Pathogenicity of the Porcine Deltacoronavirus Isolated in Southwest China. Viruses ,11 (1074), 1-22.

\begin{tabular}{|c|c|c|}
\hline & Full name & Sh \\
\hline 1 & Porcine deltacoronavirus strain USA/NorthCarolina452/2014, complete genome & $\mathrm{P}$ \\
\hline 2 & Porcine deltacoronavirus strain USA/Minnesota/2013, complete genome & \\
\hline 3 & Porcine deltacoronavirus strain USA/Illinois449/2014, complete genome & \\
\hline 4 & Porcine deltacoronavirus strain USA/Minnesota159/2014, complete genome & \\
\hline 5 & Deltacoronavirus PDCoV/USA/Illinois134/2014 from USA, complete genome & \\
\hline 6 & Porcine deltacoronavirus strain USA/Nebraska210/2014, complete genome & \\
\hline 7 & Porcine deltacoronavirus genomic RNA, complete genome, strain: YMG/JPN/2014 & \\
\hline 8 & Porcine deltacoronavirus strain USA/Michigan448/2014, complete genome & \\
\hline 9 & Porcine deltacoronavirus strain USA/Michigan447/2014, complete genome & \\
\hline 10 & Deltacoronavirus PDCoV/USA/Ohio137/2014 from USA, complete genome & \\
\hline 11 & Porcine deltacoronavirus strain USA/Indiana453/2014, complete genome & \\
\hline 12 & Porcine deltacoronavirus strain USA/Minnesota455/2014, complete genome & \\
\hline 13 & Porcine deltacoronavirus strain USA/Minnesota454/2014, complete genome & \\
\hline 14 & Porcine deltacoronavirus strain USA/Arkansas61/2015, complete genome & \\
\hline 15 & Porcine deltacoronavirus strain SHJS/SL/2016, complete genome & \\
\hline 16 & Porcine deltacoronavirus strain $\mathrm{CH} / \mathrm{SXD} 1 / 2015$, complete genome & \\
\hline 17 & Porcine deltacoronavirus strain CHN-LYG-2014, complete genome & \\
\hline 18 & Porcine deltacoronavirus isolate PDCoV/CHJXNI2/2015, complete genome & \\
\hline 19 & Porcine deltacoronavirus isolate $\mathrm{CHN}-\mathrm{AH}-2004$, complete genome & \\
\hline 20 & Porcine deltacoronavirus strain PDCoV/Swine/Vietnam/HaNoi6/2015, complete genome & \\
\hline 21 & Porcine deltacoronavirus strain PDCoV/Swine/Vietnam/Binh21/2015, complete genome & \\
\hline 22 & Porcine deltacoronavirus strain PDCoV/Swine/Thailand/S5015L/2015, complete genome & \\
\hline 23 & Porcine deltacoronavirus strain PDCoV/Swine/Thailand/S5011/2015, complete genome & \\
\hline $24^{+}$ & Porcine deltacoronavirus isolate YUC/UICMPR/2015 spike glycoprotein (S) gene, complete cds & \\
\hline $25^{+}$ & Porcine deltacoronavirus isolate MEXICO/OAX/UI1253CMPR/2017 spike glycoprotein (S) gene, complete cds & \\
\hline $26^{+}$ & Porcine deltacoronavirus isolate EDOMEX/UI202CMPR/2017 spike glycoprotein (S) gene, complete cds & \\
\hline $27^{+}$ & Porcine deltacoronavirus isolate QRO/UI689CMPR/2017 spike glycoprotein (S) gene, complete cds & \\
\hline
\end{tabular}

Table 1. Porcine Deltacoronavirus nucleotide sequences used for phylogenetic analysis 
+ Nucleotide sequences included for phylogenetic analysis of S gene.

Table 2. Genetic distances of PDCoV nucleotide sequences (percentages) with the Peruvian PDCoV strains

\begin{tabular}{lllllllll}
\hline & Whole genome & ORF $1 a / b$ & $S$ & $E$ & $M$ & $N S 6$ & $N$ & $N$ \\
\hline USA & $99.45-99.51$ & $99.56-99.63$ & $99.15-99.30$ & 99.6 & $99.38-99.85$ & $99.29-99.65$ & $99.21-99.61$ & 98 \\
China & $98.60-98.74$ & $98.72-98.86$ & $97.65-98.38$ & $98.36-98.78$ & $98.26-99.07$ & $98.93-99.29$ & $98.10-98.91$ & 98 \\
Japan & 99.5 & 99.61 & 99.24 & 99.6 & 99.85 & 99.29 & 99.51 & 99 \\
Thailand & $97.10-97.11$ & $97.27-97.28$ & $95.51-95.54$ & 99.19 & 98.26 & 98.57 & 96.83 & 97 \\
Vietnam & $97.46-97.5$ & $97.50-97.53$ & $95.95-96.05$ & 99.6 & 98.91 & 98.93 & 98.61 & 98 \\
Mexico & - & - & $98.65-99.21$ & - & - & - & - & - \\
\hline
\end{tabular}

Figure legends

Figure 1. PDCoV isolation in PK-15 cells and geographical map indicating locations where the outbreak was reported

Following PDCoV detection, intestinal content was filtered, and TPCK-trypsin treated for isolation in cell lines known to be permissible for PDCoV replication. Following 5 days of inoculation, no cytopathic effect (cpe) was evidenced under light microscopy in both cell lines. A second passage evidenced cpe after $24 \mathrm{~h}$, with initial cell rounding and monolayer disruption in PK-15 cells but absent in Vero cells. Following $48 \mathrm{~h}$ of second passage, cpe was evidenced in almost $70 \%$ of cell monolayer, characterized by cell rounding, cell detaching, pyknosis in PK-15 cell lines but no cytopathic effect was detected in Vero cell lines (A). Moreover, an immunofluorescence test with a PDCoV nucleoprotein monoclonal antibody confirmed viral replication in PK-15 infected cells (B). A representative map of South America (blue) highlighting Peru (orange) is shown in the upper right side. Additionally, a geographical map of San Martin department (yellow) located in the north of Peru (light green) is represented below (C).

Figure 2. Genomic organization of the Peruvian PDCoV isolate.

Whole genome sequence of the Peruvian PDCoV isolate was performed by next generation sequencing (NGS). PDCoV whole genome sequence is 25501 nt. Starting from 5' end, the genome is structured as follows: 5'UTR, ORF 1a/b, S, E, M, NS6, N, NS7 and 3'-UTR (A). Genomic regions, nucleotide sequence range, and nucleotide and protein lengths are depicted at the bottom (B).

Figure 3. Whole genome and ORF 1a/b phylogenetic analysis of Peruvian PDCoV strain reveals its evolutionary origin from a North American PDCoV strain

For both whole genome and ORF $1 \mathrm{a} / \mathrm{b}$, the evolutionary history was inferred by using the Maximum Likelihood method and General Time Reversible model. The percentage of trees in which the associated taxa clustered together is shown next to the branches. A discrete Gamma distribution was used to model evolutionary rate differences among sites. The tree is drawn to scale, with branch lengths measured in the number of substitutions per site. This analysis involved 24 nucleotide sequences. Evolutionary analyses were conducted in MEGA X.

Figure 4. Phylogenetic analysis of $\mathrm{S}$ gene reveals that Peruvian PDCoV strain has a close relationship with a Mexican strain within the North American phylogroup.

The evolutionary history was inferred by using the Maximum Likelihood method and General Time Reversible model. The percentage of trees in which the associated taxa clustered together is shown next to the branches. A discrete Gamma distribution was used to model evolutionary rate differences among. The tree is drawn to scale, with branch lengths measured in the number of substitutions per site. This analysis involved 28 nucleotide sequences. Evolutionary analyses were conducted in MEGA X (A). Schematic representation of the Porcine Deltacoronavirus S protein highlighting the amino acid substitutions found in the present 
study. Black arrows represent the site of these substitutions. A multiple amino acid sequence alignment of $\mathrm{S}$ protein was performed using ClustalW in MEGA X. Eight amino acid modifications were detected within the Peruvian sequence compared to others (B).

Hosted file

Deltacoronavirus article figures.pptx available at https://authorea.com/users/329914/ articles/545731-first-isolation-and-whole-genome-characterization-of-porcine-

deltacoronavirus-from-pigs-in-peru 